\title{
Papel de la reeducación funcional en piscina: nuestra experiencia en los balnearios de Caldas de Boí y Lanjarón
}

\author{
Lorena VELA ${ }^{(1)}$, Albano CHAVERO ${ }^{(1)}$ \\ ${ }^{(1)}$ Servicio Médico, Balneario de Caldas de Bohí, C/ Afueras s/n, 25528 - Caldes de Boí, \\ Lleida, Cataluña (España). \\ mlorenavela@gmail.com
}

Recibido: 15-07-11

Aceptado: 09-09-11

\section{Resumen}

La salud se ha convertido en uno de los temas que preocupan a la sociedad de nuestros días, y como consecuencia surgen temas de interés relacionados directamente con los programas de salud: el cuidado del cuerpo, la mejora de la calidad de vida, vivir una vida de forma sana y autónoma. Sin duda alguna, la salud siempre ha estado asociada a la mejoría a través del agua, es por ello que en el siguiente trabajo se establece una relación directa entre estos dos términos.

La propuesta que se expone a continuación pretende describir la implementación de la reeducación funcional en los Balnearios de Lanjarón (Granada, España) y Caldes de Boí (Lérida, España), utilizando el medio acuático como principal recurso.

También se pretende describir los diferentes programas acuáticos como son: Método Halliwick, Método de los anillos de Bad Ragaz, Relajación acuática asistida y Gimnasia acuática asistida.

Palabras claves: Reeducación funcional, Halliwick, Ai-Chi, Bad Ragaz, Relajación acuática asistida, Gimnasia acuática terapéutica.

Role of functional rehabilitation in the pool: our experience at the Spas Caldas de Boí and Lanjarón.

\begin{abstract}
Health has become one of the issues that concern society today, and as a result of interest issues arise directly related to health programs: body care, improving quality of life, living a life a healthy and independent. Health has always been associated with the aquatic environment, which is why the next job in establishing a direct relationship between these two terms.

The proposal outlined below is intended to describe the implementation of functional recovery Spas in Lanjarón (Granada, Spain) and Caldes de Boí (Lleida, Spain), using the aquatic environment as the main resource.
\end{abstract}


It also aims to describe the different aquatic programs including: Halliwick Method, Method of rings Bad Ragaz, Water-assisted relaxation and assisted Water aerobics.

Key words: Functional Rehabilitation, Halliwick, Ai-Chi, Bad Ragaz, Water assisted relaxation, Aqua therapy

REFERENCIA NORMALIZADA

Vela L, Chavero A. Papel de la reeducación funcional en piscina: nuestra experiencia en los balnearios de Caldas de Boí y Lanjarón. (Role of functional rehabilitation in the pool: our experience at the Spas Caldas de Boí and Lanjarón). Anal Hidrol Med, 2011, vol. 4, 45-56

\section{INTRODUCCIÓN}

La reeducación funcional es, sin duda, un medio valioso y de gran importancia en los centros balnearios como terapia coadyuvante en la prevención y recuperación funcional de alteraciones del aparato locomotor, de tipo reumático, postraumático o neurológico, problemas frecuentes en las personas que acuden al balneario.

Debemos hablar de una forma de terapia física que nace en el año 1960 de la mano de los noruegos Gustavson y Evjenth, estos expertos pasaron a eliminar las largas fases de inmovilización y como solución desarrollaron el tratamiento activo, la cinesiterapia activa. Aparte de las medidas pasivas de tratamiento como fangoterapia, baños de inmersión, masajes, ducha circular, que encontramos en los centros balnearios, es muy importante que este tipo de terapia sea parte de los tratamientos que se ofrecen ${ }^{1}$.

\section{TERAPIA FÍSICA EN EL MEDIO ACUÁTICO}

Aunque el agua se haya usado terapéuticamente durante siglos, sólo en los últimos tiempos se ha extendido su empleo en la reeducación funcional. Las propiedades de la flotabilidad y resistencia del agua la convierten en una herramienta útil para los especialistas en reeducación ${ }^{2-3-4-5-6-7-8-9}$.

Las ventajas de rebajar la fuerza de la gravedad y de la inmersión en un medio resistido son por todos conocidas, y el empleo del agua como medio rehabilitador se ha ido extendiendo.

El medio acuático nos brinda la posibilidad de aplicar diferentes técnicas, poniendo especial valor en el Método Halliwick, Método de los Anillos de Bad Ragaz, Ai-Chi, Relajación Acuática Asistida y Gimnasia Acuática Terapéutica, terapias que sin duda le pueden dar un valor agregado a la cura balnearia.

¿En qué consisten cada uno ellos? 


\section{Halliwick:}

Es un concepto originalmente desarrollado para enseñar a pacientes con discapacidad física a nadar y a ser independientes en el agua. El concepto fue fundado por James McMillan, un ingeniero de mecánica de fluidos. Sus objetivos principales eran la participación y la independencia.

El Programa de diez puntos se utiliza para llegar a estos objetivos. La parte más importante de este programa es el control rotacional. También es básico para una segunda parte del concepto Halliwick: la Terapia Específica en el Agua (WST). Esta parte está centrada en el control postural, normalizando la rigidez muscular y facilitando el movimiento. Halliwick se usa principalmente en neurorehabilitacion y pediatría. Tiene mucha semejanza con el método Bobath que se aplica en seco.

Las posibilidades y limitaciones del paciente son analizados con el fin de utilizar una intervención sistemática (Programa de diez puntos y / o WST) para ayudar a los pacientes aumentando la ganancia funcional.

Las ventajas mecánicas del agua dan soporte a las habilidades del tronco movilizándolo y estabilizándolo. En este sentido, Halliwick es una terapia de movimiento inducido por restricción sin la desventaja de compensación de la gravedad. Muchas actividades pueden ser repetidas y variadas y los pacientes pueden aprender estrategias de equilibrio -y desequilibrio-, las cuales pueden traspasar a la tierra firme.

Halliwick también permite un programa de actividades clasificadas de bajo impacto mecánico y creciente demanda fisiológica; los pacientes con dolor crónico de espalda y otros pueden aumentar su capacidad funcional de una forma sobre todo lúdica $^{10-11-12}$.

\section{Método de los anillos de Bad Ragaz:}

El Método de los Anillos de Bad Ragaz (Bad Ragaz Ring Method, BRRM) es una técnica activa en la que el terapeuta ofrece resistencia manual al paciente, con el apoyo de materiales auxiliares de flotación. Al paciente se le facilita, sobre todo propioceptivamente, para activar los músculos débiles.

BRRM sigue los principios de Facilitación Neuromuscular Propioceptiva (FNP), pero está adaptado a las posibilidades y dificultades de moverse en un ambiente acuático. BRRM consta de unos 23 patrones de brazos, tronco y piernas. En varios patrones se pueden utilizar técnicas de FNP como por ejemplo la combinación de isotónicos. Aparte de eso, la fisiología de fortalecimiento muscular se ha incluido en la última década.

Los principales objetivos de BRRM son activar los músculos débiles a través de los principios de la irradiación segmentaria, aumentando la fuerza muscular y la estabilización muscular de las articulaciones. El BRRM por lo tanto se limita al 
nivel de función de la CIF, y está dirigido a una tarea concreta o un objetivo orientado $^{13}$.

\section{Ai-Chi:}

Ai-Chi es un programa de ejercicios acuáticos y de relajación que combina la respiración profunda con movimientos amplios y lentos, continuos y fluidos realizados según unos patrones preestablecidos. El Ai Chi fue creado por el japonés Jun Konno.

Es una forma de fitness acuático que se concentra principalmente en el equilibrio, la fuerza, la relajación, la flexibilidad y la respiración. Es como una meditación en movimiento que trae equilibrio a nuestro cuerpo y a nuestra mente. Generalmente se realiza en grupos dentro de la piscina. Los lentos y amplios movimientos de brazos y piernas, junto con la respiración y una música suave para facilitar la relajación, ayudan a estimular y a que fluya la energía interna.

El Ai Chi es un trabajo que llega al cuerpo, a la mente y al espíritu y que nos ayuda tanto físicamente como en nuestras actividades diarias.

\section{Relajación Acuática Asistida:}

Es un método de terapia acuática integral, tendiente a restablecer el equilibrio físico-mental y emocional. En esta terapia se aprovechan los beneficios y las propiedades que tiene el agua caliente, ofreciendo efectos positivos tanto físicos como psíquicos, permite además que el cuerpo en el agua pueda adoptar diferentes posiciones ya sea con la sujeción del terapeuta, que es el punto de referencia para movilizar a la persona, o asistido por flotadores especiales, lo que permite poder realizar un trabajo corporal sin riesgos. Para ello se utilizan diferentes movimientos de elongación, masajes y también distintos tipos de movimientos de grupos musculares, permitiendo además actuar alrededor de todos los ejes de aquellas articulaciones con problemas.

Esta terapia también ofrece una alternativa diferente en los distintos problemas de la columna vertebral, con técnicas que no se realizan en forma agresiva, sino suavemente y en forma progresiva, puesto que en el agua se puede movilizar la columna y articulaciones de una manera mucho más fácil y placentera que si lo realizáramos fuera de este medio.

Unos de los objetivos de esta terapia es también llevar a la persona a una relajación muscular para disminuir la estimulación cerebral superior y consciente, cortical, produciendo un estado de bienestar y calma, ya que un aumento de la tensión muscular produce un aumento de tensión cerebral y al contrario una disminución de la tensión muscular conlleva a una disminución de la tensión psicológica. 


\section{Gimnasia Acuática Terapéutica:}

Esta consiste en la unión de los ejercicios acuáticos con la terapia física. Es un abordaje terapéutico que utiliza los ejercicios acuáticos para ayudar en la reeducación de diferentes patologías.

Cada sesión debe ser programada y organizada teniendo en consideración componentes específicos de: entrada en calor, estiramiento, fuerza muscular, resistencia aeróbica y elongación.

Esta es una actividad alternativa de nivel preventivo o correctivo que aprovechando las propiedades físicas que brinda el agua permite el trabajo corporal sin carga del peso del propio cuerpo, evitando así el impacto articular.

En el agua, los movimientos corporales sufren una resistencia al avance, que dificulta los mismos y torna los ejercicios un poco más lentos pero no menos intensos. Como las articulaciones están especialmente protegidas, no hay movimientos balísticos o descontrolados a gran velocidad.

Debido a la presión, las articulaciones sufren menos impacto durante los ejercicios, factor importantísimo para personas con condiciones posturales especiales, para obesos, personas con osteoporosis y embarazadas.

La presión hidrostática facilita el retorno venoso, mejorando la circulación y disminuye el edema, situación particularmente favorable para embarazadas y portadores de várices.

Fundamentación de la gimnasia acuática terapéutica ${ }^{14}$.

Durante las enfermedades se altera la actividad vital del organismo, disminuye su capacidad de realizar trabajo muscular de ahí que cuando los movimientos pueden originar agudización o complicación de alguna enfermedad se hace necesario guardar un régimen de reposo que limita la actividad motora del enfermo. Este régimen de reposo disminuye el consumo de oxigeno y de sustancias alimenticias por el organismo y por lo tanto contribuye a un trabajo mas económico de los órganos internos y un restablecimiento del trabajo del SNC. Sin embargo junto a estos aspectos positivos el régimen de reposo tiene aspectos negativos. Como por ejemplo se reducen los procesos de excitación en el SNC, disminuyen las posibilidades funcionales del sistema cardiovascular y respiratorio así como la nutrición del organismo: Por tales motivos es necesario cuando lo permitan las condiciones y el estado del enfermo combinar el reposo con la aplicación de ejercicios físicos. La gimnasia acuática terapéutica, por medio del ejercicio físico, disminuyen las influencias desfavorables de la actividad motora. Previene las complicaciones e intensifica las reacciones de defensa del organismo. En el período de recuperación las sesiones de gimnasia acuática terapéutica contribuyen a restituir la capacidad de trabajo. Las cargas físicas incrementadas gradualmente garantizan el entrenamiento del organismo y producen la normalización de sus funciones.

A la luz de los datos citados acerca de los mecanismos fisiológicos de la regulación de las funciones en el organismo, se hace comprensible la acción terapéutica 
de los ejercicios físicos. Esta se manifiesta en forma de 4 mecanismos básicos de acción tonificante, de acción trófica, de formación de las compensaciones y de normalización de las funciones. Para la aplicación consiente y exitosa de los ejercicios físicos acuáticos, con fines terapéuticos, es necesario conocerlos y dominarlos.

\section{BALNEARIO DE LANJARÓN}

Lanjarón es un municipio español, perteneciente a la provincia de Granada, en Andalucía. Está situado en la parte occidental de la Alpujarra Granadina, a unos 49 $\mathrm{Km}$. de la capital provincial, en el sureste de España. Gran parte de su término municipal se encuentra en el Parque Nacional de Sierra Nevada (Figura 1) ${ }^{15-16}$.

Figura 1 - Balneário de Lanjarón

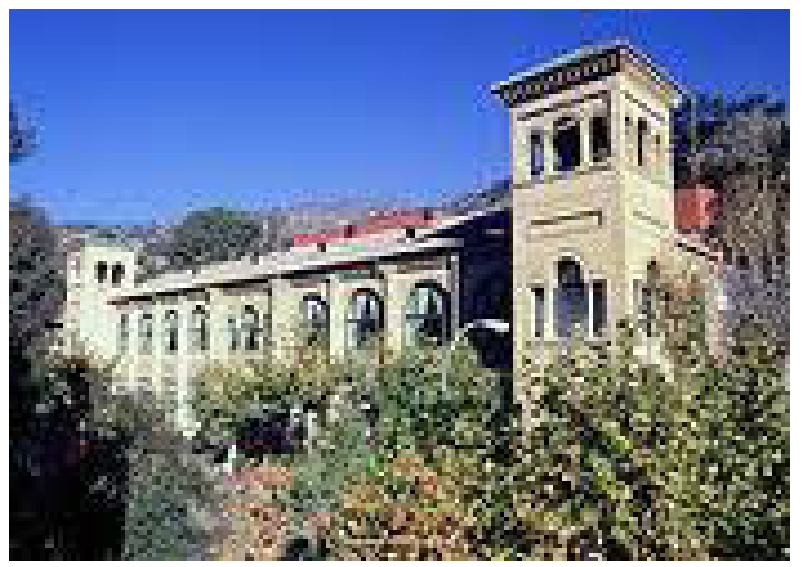

Características de las aguas ${ }^{17}$

Lanjarón-Capilla II: Facilita la digestión, Estimula el apetito, Disminuye la sensibilidad gustativa, Irrita la mucosa digestiva, Estimula la secreción y motilidad del tracto digestivo, Actividad pro-insulínica y Mejora la tolerancia a los Hidratos de Carbonos.

Lanjarón-capuchina: Estimula la secreción clorhídrica, Estimula la motilidad gástrica, Colerética y colagoga, Aumenta la secreción y motilidad intestinal, Reduce la flora intestinal, Altera el ciclo hepato/entero/hepático (colesterol), Efecto laxante y/o purgante.

Lanjarón-salado: Estimuladora de las funciones celulares, Mejoradora del trofismo tisular, Estimuladora de la actividad secretora, Activadora de las funciones 
metabólicas, Antiinflamatoria y desinfectante a nivel tópico y Modificadoras del terreno.

Lanjarón-salud II: Antiácida, Efectos antipépsicos, Efectos facilitadores de la evacuación gástrica, Efectos facilitadores de la secreción pancreática, Colagoga, Efecto sedativo y protector de la mucosa intestinal, Regula el tono vegetativo del transito intestinal y Diurética.

Lanjarón-san vicente: Aumento de la dilución plasmática, Aumento del filtrado glomerular, Descenso de la reabsorción tubular renal, Aumento del volumen y dilución de la orina, Acción mecánica de lavado y arrastre de riñón y vías urinarias, Antiséptica y espasmolítica, Acción descongestiva y sedante de las vías urinarias

\section{Indicaciones terapéuticas}

Lanjarón-capuchina, salud II y Capilla II: Afecciones gástricas que cursan con hiposecreción e hipomotilidad, Gastritis crónicas, Litiasis biliares, Discinesias biliares, Dispepsias, Convalescencias de hepatitis virales, Pequeñas insuficiencias no descompensadas, Colopatías secundarias a antibioterapia, Estreñimiento, Uso inveterado de laxantes y Diverticulosis sigmoidea.

Lanjarón-salado: Reumatismos inflamatorios, Reumatismos degenerativos, Reumatismos para-articulares, Reumatismos metabólicos y Secuelas posttraumáticas. Afecciones respiratorias.

Lanjarón-san vicente: Litiasis reno-ureterales, Infecciones urinarias y Intervenciones pre y post-litotriciales.

\section{Tratamientos}

Cura Hidropínica, Baño Normal, Baño Burbujas, Piscina Dinámica, Ducha de Chorro, Ducha Circular, Ducha Miorelajante, Maniluvio, Pediluvio, Pasillo de Cantos Rodados, Estufa de Vapor, Inhalación, Vaporización y técnicas complementarias

\section{Programa de Reeducación Funcional}

En el año 2009 se comenzó a implementar en el balneario el programa de reeducación funcional, atendiéndose diferentes patologías del aparato locomotor, cardiovascular y respiratorio (Figura 2$)^{18}$.

\section{Metodología de trabajo}

Los médicos responsables de realizar la evaluación del paciente, son lo que derivan a los mismos según la patología que presentan a la gimnasia acuática terapéutica, esta se realiza de forma grupal, donde se plantean las actividades a realizar según más convenga.

En la actualidad este programa lo llevan a la práctica dos fisioterapeutas y un monitor especializado en gimnasia acuática. 
Figura 2 - Gimnasia acuática terapéutica en el Balneario de Lanjarón
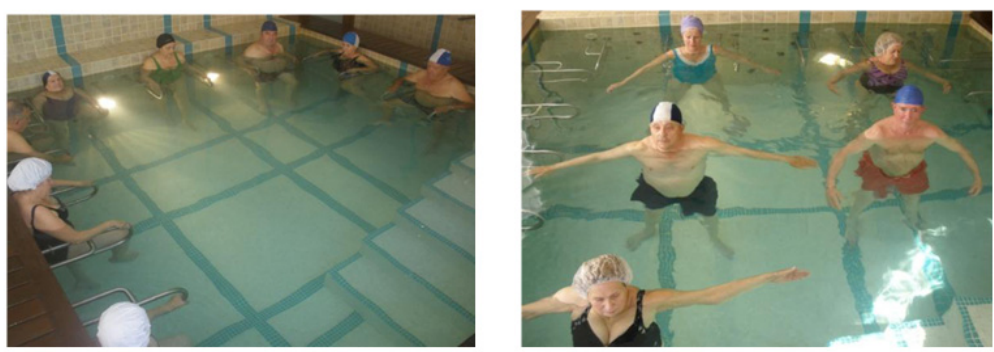

\section{BALNEARIO DE CALDES DE BOHÍ}

Caldes de Boí es un balneario de origen romano situado en el término municipal del Valle de Boí (provincia de Lérida). Se encuentra a una altura de $1.470 \mathrm{msnm}$, en la orilla del río Noguera de Tor que a esa altura recibe el nombre de río de Caldes.

El Balneario abarca 24 hectáreas de superficie, con una vasta extensión de jardines donde encontramos los 37 manantiales de aguas mineromedicinales de diferentes composiciones y temperaturas, yendo de $\operatorname{los} 4^{\circ} \mathrm{C}$ hasta $\operatorname{los} 56^{\circ} \mathrm{C}$ (Figura 3$)^{19}$.

Figura 3 - Balneario de Caldas de Boí

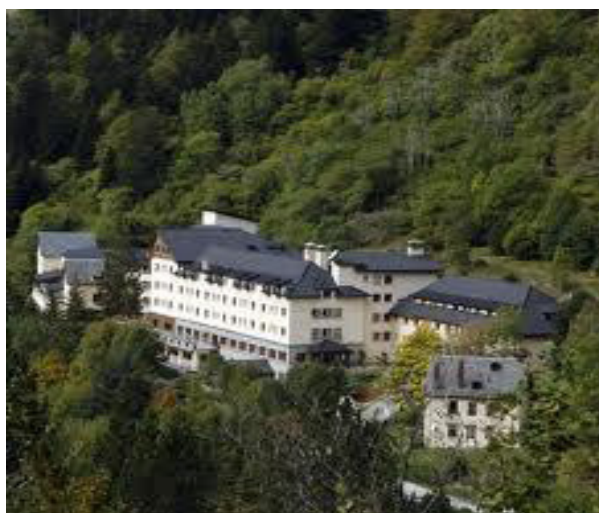

Características de las aguas e indicaciones ${ }^{20}$

La Estufa: es un manantial cuya surgencia esta dentro del centro termal. Agua hipertermal, sulfurada que emana a $50,5^{\circ} \mathrm{C}$, es el manantial más utilizado en los tratamientos del Centro Termal, el vapor de su agua se inhala en el interior de las estufas. 
Baños: este manantial emerge en el interior del Centro Termal, agua sulfurada e hipertermal que emana a $50,5^{\circ} \mathrm{C}$. Es aprovechada en el Centro Termal para diferentes tratamientos.

Tartera: una de las fuentes más preciadas y reconocidas de este Balneario, descubierta en 1849. Sulfurosa, rica en azufre, cinc y sílice, emerge a $45^{\circ} \mathrm{C}$ y está especialmente indicada para tratar afecciones de la piel mediante uso tópico y mediante su ingestión (cura hidropínica) para combatir patologías del aparato respiratorio, vías bajas.

Avellaner: agua hipotónica, bicarbonatada, clorurada, sulfatada y sódica. Muy diurética, de mineralización muy débil. Emerge a $21^{\circ} \mathrm{C}$ y su caudal es de $0,26 \mathrm{l} / \mathrm{s}$, especialmente indicada para patologías del aparato urinario, (litiasis). Facilita la eliminación de líquidos y está especialmente indicada en programas de adelgazamiento y anticelulíticos.

Bou: este manantial se comercializa para consumo doméstico con el nombre de Agua de Boí. Emerge a $36^{\circ} \mathrm{C}$ de temperatura, con un caudal de 3,6 1/s, es un agua mesotermal, de mineralización muy débil, hipotónica, blanda, clorurada, bicarbonatada y rica en sílice, esta indicada para problemas de gastritis, gota y litiasis renal.

Bosch: agua mesotermal, de mineralización muy débil, sulfurada-cálcicosulfhídrica, emana a $30,5^{\circ}$ C. Está especialmente indicada para la insuficiencia hepática, para tratar trastornos del aparato digestivo o digestiones pesadas. Su ingestión ayuda a regular el índice de colesterol.

Canem: agua sulfurada, mesotermal, emana a $38,5^{\circ} \mathrm{C}$, es un agua mesotermal de mineralización muy débil, se aplica en piscinas y en el centro termal.

\section{Tratamientos}

Circuito termal, Inhalaciones, Aerosoles, Estufas, Masaje terapéutico de espalda, Vaporariums, Ducha circular, Fangoterapia, baños de Tartera, Ducha Vichy, Masaje subacuático.

\section{Programa de Reeducación Funcional}

En el año 2010 se pone en marcha el programa de Reeducación funcional y Salud, el cual se conforma con actividades tanto acuáticas como en seco.

El mismo está a cargo de un licenciado en Actividad física, especializado en hidrocinesioterapia y readaptación física.

Actividades acuáticas (Figura 4 y 5):

a) Método Halliwick.

b) Método de los anillos de Bad Ragaz.

c) Ai-Chi.

d) Gimnasia acuática terapéutica.

e) Relajación acuática asistida 
Figura 4 - Sesión de hidrocinesioterapia

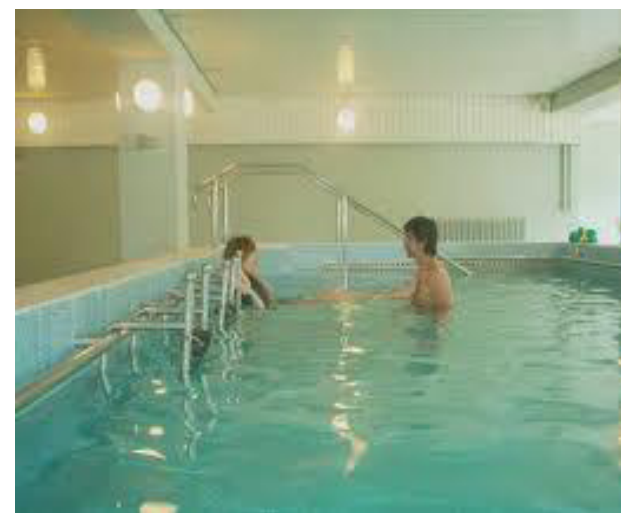

Figura 5 - Sesión de Halliwick

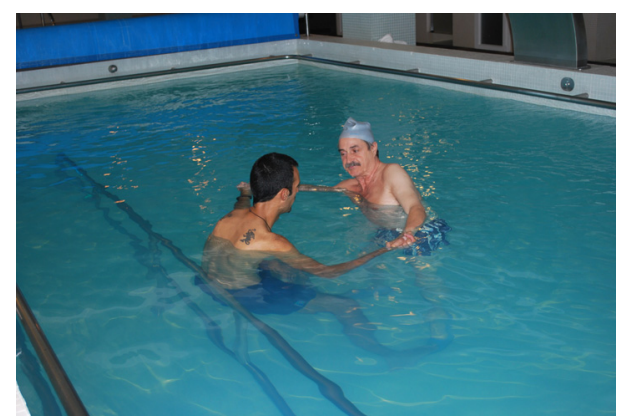

\section{Metodología de trabajo}

En el balneario Caldes de Boí el médico responsable de realizar la evaluación inicial del paciente recomienda a que actividad puede acogerse teniendo en cuenta que patología presenta y a la que se quiere dar atención. Luego el profesional que lleva adelante los diferentes programas, será el responsable de seleccionar las actividades que mejor se adapten al paciente, siempre basado en las observaciones hechas por el médico.

Una vez finalizado el tratamiento termal el medico vuelve a realizar la evaluación final del paciente.

Estas diferentes actividades son también parte complementaria de paquetes de tratamientos, añadiéndole un valor a los mismos. 


\section{CONCLUSIÓN:}

Para concluir se puede destacar que en el Balneario de Lanjarón en el año 2009 se realizó una encuesta con el objetivo de valorar el programa de gimnasia acuática. Del total de los encuestados el 51\% realiza ejercicios habitualmente, el $94 \%$ manifiesta cambios positivos después de haber realizado las 5 sesiones de gimnasia acuática, el 67\% refiere tener la intención de continuar con esta actividad en su lugar de residencia, el 100\% de los encuestados valora positivamente la actividad y expone que se debería realizar en todos los balnearios de España. Sólo un $11 \%$ había realizado gimnasia acuática en otro balneario. El 88\% tiene la intención de contratar el programa el próximo año.

En el Balneario Caldes de Boí la implementación del programa de Reeducación funcional, ha provocado una excelente respuesta en los usuarios: el $80 \%$ de los pacientes que concurre a través del programa de Imserso contratan alguna de las opciones y el $95 \%$ queda altamente satisfecho. Un $80 \%$ de los pacientes que realizan las terapias mas innovadoras como son el Ai-chi, Bad Ragaz, Halliwick y la relajación acuática asistida vuelven para repetir la terapia.

\section{BIBLIOGRAFÍA}

1. Maraver F. Importancia de la medicina termal. Balnea 2008; 4: 35-50.

2. Bates A, Hanson N. Ejercicios acuáticos terapéuticos. San Pablo: Manole, 1998.

3. Freese J. Fitness Terapéutico. Badalona: Paidotribo, 2006.

4. Hall C, Brody L. Ejercicio Terapéutico, recuperación funcional. Badalona: Paidotribo, 2006.

5. Koury JM. Acuaterapia. Guía de rehabilitación y fisioterapia en la piscina. Barcelona: Bellaterra, 1998.

6. Becker BE, Cole AJ. Comprehensive aquatic therapy. Boston: ButterworthHeinemann, 1997.

7. Meijide R, Rodríguez-Villamil JL, Teijeiro J. Hidroterapia. En: MartínezMorillo M et al. Manual de Medicina Física. Barcelona: Harcourt, 2000, 335357.

8. Pérez MR. Principios de hidroterapia y balneoterapia. Madrid: Mc Graw-Hill, 2005.

9. San Martin, J. Técnicas actuales de tratamiento balneario. Balneocinesiterapia. En: López-Geta JA, Pinuaga JI (editores). Panorama actual de las Aguas Minerales y Minero-medicinales en España. Madrid: ITGE, 2000, 105-114.

10. Reid MJ. Activity in water based on the Halliwick method. Child Care Health Dev. 1975 Jul-Aug;1(4):217-23.

11. Martin J. The Halliwick Method. Physiotherapy. 1981 Oct;67(10):288-91. 
12. Red Internacional de Terapia Halliwick. Método Halliwick. 2011. Disponible en: http://www.halliwicktherapy.org/es.html.

13. Fundación del Método de los Anillos de Bad Ragaz. Método de los anillos de Bad Ragaz. 2011. Disponible en: http://www.badragazringmethod.org/es.html

14. Red Española de terapia acuática, técnicas. 2011. Disponible en: http://www.retacua.org/.

15. Albiol V. Perfil sociosanitario de la población balnearia beneficiaria del programa de termalismo social del establecimiento balneario de Lanjarón (Granada) [tesis]. Madrid: Universidad Complutense, 1999.

16. Piñar Samos J (coord.). Lanjarón. Paisajes del Agua. Granada: BALSA, 1999: $317 \mathrm{p}$.

17. Maraver F, Armijo F. Balneario de Lanjarón. En: Maraver F, Armijo F. Vademécum II de aguas mineromedicinales españolas. Madrid: Complutense, 2010: 74-79.

18. Vela L, Chavero A, Rodríguez P, Platero JA. Valoración por los usuarios del programa de gimnasia acuática desarrollado en el Balneario de Lanjarón. Granada. Bol Soc Esp Hidrol Med. 2010; 25; 77.

19. ValeroA A.: Estación Termal Caldes de Boi. Madrid: Gráficas Aras Montano S.A., 1990.

20. VVAA. Balneario de Caldas de Bohi. An Real Acad Farm. 1989;15: 115 p. 